\title{
Clinical assessment of Nociceptive trigeminal inhibition tension suppression system (NTI-tss) appliance for Temporomandibular joint disorder associated with headache- A Case report
}

\author{
Ravi M B ${ }^{1}$, Sowmya $\mathrm{S}^{2}$, Akansha Roy ${ }^{3}$ \\ ${ }^{1}$ Reader, ${ }^{2}$ Assistant Professor, ${ }^{3}$ Post Graduate \\ Department of Prosthodontics, JSS Dental College and Hospital, JSS Academy of Higher Education and Research, Mysuru, India \\ Correspondence: sowmya.neelan@gmail.com
}

\begin{abstract}
Temporomandibular disorder and bruxism may cause individuals to experience extreme pain in the region of jaw and head. The associated musculature and the joint are placed at a strained position which manifests as unbearable pain. This pain may radiate to face, neck and some may even develop migraine, all of which can have painful and a debilitating effect on an individual. This paper describes the case where a patient who was suffering from TMJ pain from 8 years using multiple occlusal splints over the course of years but showed no signs of relief thus, impairing the quality of life. TMD's constitute a group of disease with overlapping similar sign and symptoms. In this case, the patient was diagnosed and treated with the NTI-tSS appliance, with a 6 month follow up showing almost complete resolution of the symptoms enhancing the quality of life.
\end{abstract}

Key Words: NTI-tss, TMD, Myofacial pain

\section{Introduction}

Temporomandibular disorders (TMD) comprises of various clinical issues faced with respect to any muscles of mastication, the temperomandibular joint or any related structures.[1] Pain in the masticatory muscle presents as the most observed symptom associated to TMD. [2-4]Though, any patient with TMD generally reports with a common complaint of jaw pain, ear pain, clicking sound in the jaws while opening or closing, overall pain in the face, and severe headache.[3] De Rossi et al., established a significant and a pivotal relationship of TMD and headache, showing a significantly higher prevalence of TMD in patients presenting to a neurology clinic for headache than a control population.[5]

Almost three billion individuals were estimated to have a migraine or tension-type head ache[6] Globally, it has been estimated that prevalence among adults of current headache disorder (symptomatic at least once within the last year) is about $50 \%$ migraine and severe tensiontype headaches are not limited to adults. The prevalence in children increases from $39 \%$ at age 6 to over $70 \%$ by age 15.[7-8] In addition, more than one million days of missed school can be directly attributed to these types of headache pain.[6]

In this paper we intend to discuss a case report and the management of TMD's with severe headache and jaw pain as the primary symptom showing no relief with any occlusal splint and post first line of therapy with a longer duration of symptoms since years without any relief thus leading to a very worsen quality of life.

\section{Case Report}

A 22 year old, male patient reported to the Department of Prosthodontics, Crown and Bridge in the JSS Dental College and Hospital, Mysuru with the chief complaint of constant pain in the jaws since past 8 years, aggravating on opening the mouth further leading to headache on extended duration. Patient had a history of deviation of jaws with self-guidance to its original position. The patient also had a history of bruxism especially in the anterior teeth. Upon which the patient also reported of using multiple occlusal splints (5 in total) over the course of 4 years with eventual perforation of the occlusal splint (hard and soft ) at the point of high contact near the 18 and 48 region Fig 1. Additionally the patient complained about the episodes of headaches in the morning with tenderness in the muscles of mastication upon waking up.

On Extra Oral examination of the TMJ, there was a bilateral clicking noted while opening of the jaws, deviation was noted towards the right side while closing the mouth. No muscle tenderness was noted at the moment. 
Though, occasional bilateral submandibular lymph node ( Level II) were palpable.

On Intra oral examination, teeth missing were only 28 and 38 due to the history of extraction. Incisal wear facets were noted in the maxillary and the mandibular combining anterior twelve teeth. Increased over bite was noted.

Diagnostic impressions were made and casts were poured with dental stone to initiate the treatment plan. NTI- Tss appliance was decided to be fabricated considering the symptoms for the management. Bite registration was done with modelling bite registration wax and facebow transfer Fig. 2,3. was done for the patient to establish the condylar and the incisal guidance and simulate it outside the patient's mouth on a semi adjustable Hanau wide vue articulator. The vertical dimension was established. incisors, thereby increasing the vertical dimension between the upper and lower jaw.[5] Adjustments along the outer surface of the bite stop are made by the clinician to ensure that at jaw closure and during excursive

movements tooth contacts are present only between the intraoral device and the incisal embrasures of the antagonistic teeth Fig. 4 This "anterior bite appliance" is though typically worn during the night, although two variations of the bite stop are offered for daytime use.[10]

\section{Discussion}

Myofascial pain is the most prevalent disorder among TMDs, accounting for more than half of the cases. [11] The site of pain is generally located in the temple region and muscles of mastication which may also extend far from its site of origin.[7] In addition to pain, limitation of

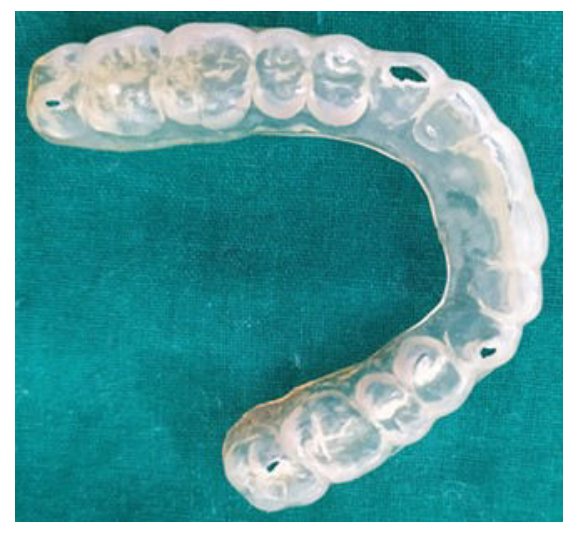

Fig.1. Previous Occlusal Soft

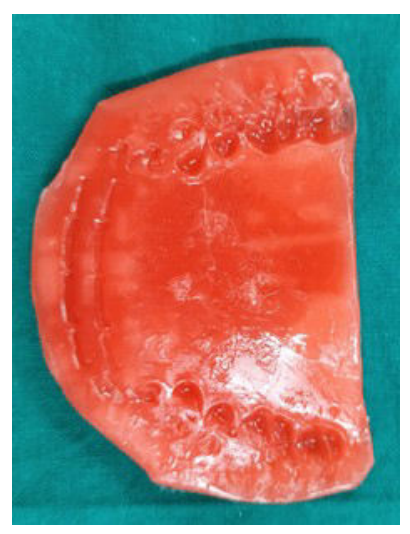

Fig.2 Bite registration

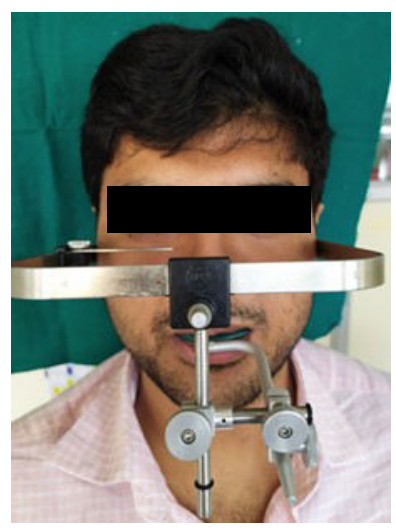

Fig.3.Face bow Transfer

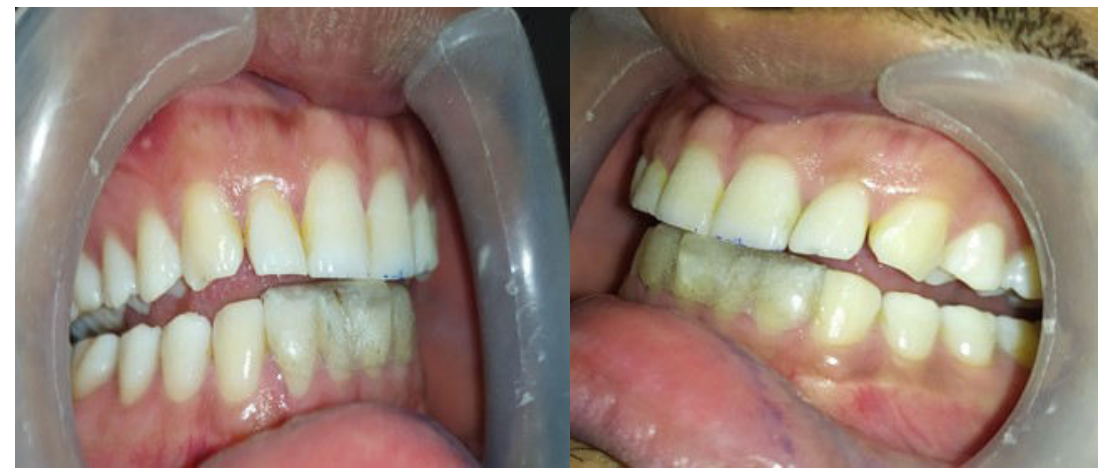

Fig.4. Adjustment of NTI-tss appliance

To fabricate the NTI- tss device which is primarily a small pre fabricated anterior bite stop which covers in its most widely used form - the two maxillary (or mandibular) central incisors.[9] The fit along the teeth is accomplished at the chair side by filling either an auto-polymerizing acrylate or a thermoplastic material into the base of the device, which is subsequently adapted along the central jaw movement may be a burden for the patient.[12] The etiology of myofascial pain is diverse, but multiple factors such as direct or indirect macro traumatic events, oral para functional habits, postural disturbances and psychosocial stressors have been implicated.[13] Additionally, bruxism, as a movement disorder often associated with sleep, has been suggested as a predominant factor in the onset or 
continuance of myofascial pain.[7]

The management of myofascial pain is generally directed at relieving symptoms and enhancing the quality of life.[14] Identification, assessment and elimination of precipitating and of life.[14] Identification, assessment and elimination of precipitating and perpetuating factors are important parts of the management of myofascial pain.[2] Various manage elimination of precipitating and perpetuating factors are important parts of the management of myofascial pain.[2] Various management techniques, mostly conservative and non-invasive, have been suggested.[15] A recent systematic review pointed out the beneficial effects of cognitive-behavioural therapy and self-care management.[12,16] Different school of thoughts suggest various mechanisms to deal with such a common but highly debilitating issue faced as the most often disease after dental caries.[17] Correcting the occlusal equilibration plays the significant part in the management of TMD's.[18] Disocclusion or any high contact during occlusion creates an imbalance in the relaxation of the masticatory muscles considering their role in maximal intercuspation and in centric relation.[19] The relieve of the high contacts to attain an occlusal equilibration helps in relieving the symptoms the TMD's.[7,20]

In addition, intra-oral splints either alone or in combination with other approaches are often used to reduce pain and improve function in patients with myofascial pain.[1,15,16,21 ) Among different types of occlusal splints, stabilization splints and nociceptive trigeminal inhibition splint have been reported as safer and more effective than splints with irreversible designs.[22-24] Nociceptive trigeminal inhibition tension suppression system (NTItss) splint is a pre-fabricated device with extremely small dimensions that covers only two maxillary or mandibular central incisors.(25) For its adjustment, the thermoplastic material provided in the box with the splint is melted in hot water, filled into the concave region of the splint and adapted to lower or upper incisor teeth.[21] The material re -polymerises again, becomes rigid, fits to the anterior teeth and avoids contact of canines and molars. This splint was developed to trigger the jaw-opening reflex, which protects teeth from excessive forces.[5,10,25] Although guidance, assurance, counselling and behavioural changes are recommended as first-line therapy.[12,17,16]

1. Mechanism of Action: NTI-tss allows for optimum musculoskeletal stability in the condylar position. $[5,10,25]$ If a patient's condylar position is not optimal, the condyle may re-position more posteriorly/ superiorly during resolution of symptoms.[27]
In June 2001, the FDA approved the use of NTI-tss for the following indications:

1. A device to be used in the prophylactic treatment of medically diagnosed migraine pain as well as migraineassociated tension-type headaches by reducing their signs and symptoms through reduction of trigeminally innervated muscular activity [8]

2. For prevention of bruxism and TMJ syndrome through reduction of trigeminally innervated muscular activity. [16]

Although guidance, assurance, counselling and behavioral changes are recommended as first-line therapy. $[12,17,16]$

In a systematic review, Stapelmann and Teurp reported that NTI-tss splint may be successfully used for management of TMDs and bruxism.[10] However, they added that the use of this device may be accompanied by unwanted effects.[10] Compared with stabilization splints, NTI-tss splints do not need fabrication of casts, may need a shorter adjustment time and are small in dimensions, which makes these a choice of therapy for both clinicians and patients.[25,27,28]

Post usage of the appliance for three weeks, there was a significant reduction noted in the episodes of migraine from 2 genuine episodes in 10 days of extending upto a diurnal duration to only one small episode of lasting maximally upto an hour. Intermediate clicking was noted upon the usage of three weeks with hyperactivity of temporalis. The patient showed great relief within the usage of the appliance of minimal three weeks. The patient was followed up consecutively for the check of the symptoms. On 6 months follow up, the patient had a significant improvement in the pain management and $a b-$ sence of clicking was noted in the TMJ while performing the movements of the mandible.

\section{Conclusion}

Myofascial pain dysfunction syndrome has always been a point of conundrum for all the clinicians and especially its treatment. Each case has to be dealt subjectively with its presenting symptoms. NTI-tss reports to have added advantages in cases pertaining to TMJ pain associated with migraine headache. Further studies are needed to generate a protocol for an easier approach towards the treatment.

\section{References}

Maia T, Fernandes BB, Reis J, Berlanga T, Soffener DA, Bernardes VL, et al. Evaluation of psychosocial aspects 
in patients with TMD: Catastrophic pain, anxiety and depression - Literature review. 2017;07(10):17425-31.

2. Schiffman E, Ohrbach R, Truelove E, Look J, Anderson G, Goulet J-P, et al. Diagnostic Criteria for Temporomandibular Disorders (DC/TMD) for Clinical and Research Applications: Recommendations of the International RDC/TMD Consortium Network and Orofacial Pain Special Interest Group $\dagger$. J Oral Facial Pain Headache. 2014;28(1):6-27.

3. Newcastle School of Dental Sciences - TMD Research Diagnostic Criteria. 2003;1: 6-7

4. Costa, Abreu, Cendes F. Temporomandibular joint internal derangement Association with headache, joint effusion, bruxism, and joint pain. J. Contemp. Dent. Pract .2008; 9:009-016

5. Srivastava R. NTI-tss ${ }^{\circledR}$ - A Novel Approach To The Management Of Temporomandibular Joint Disorders. 2014;(March).

6. Lars Jacob Stovner, Global, regional, and national burden of migraine and tension-type headache,19902016: a systematic analysis for the Global Burden of Disease Study 2016. Lancet Neurol,2018 17,954-976

7. Anthony Kilcoyne, Dental occlusion problems are a major cause of headache BMJ 2017;345:29-30

8. Shankland W, Migraine and Tension-Type Headache Reduction Through Pericranial Muscular Suppression: A Preliminary Report. The Journal of Craniomandibular \& Sleep Practice2001; 19:269-278.

9. Fe, R. S., Examiner, P. Desanto, M. F. (12) United States Patent. 2, (2010).

10. Stapelmann H, Türp JC, The NTI-tss device for the therapy of bruxism, temporomandibular disorders, and headache - Where do we stand? A qualitative systematic review of the literature.BMC Oral Health.2008;8-22

11. Raphael KG, Marbach JJ. Widespread pain and the effectiveness of oral splints in myofascial face pain. J. Am. Dent. Assoc 2001; 132:305-316.

12. Nandhini J. Nonsurgical management effective in temporomandibular joint disorders? -A systematic review and meta-analysis. Dent. Res. J. (Isfahan).2018; 15:231-241.

13. Graff-Radford, Steven B. Temporomandibular disorders, and other causes of facial pain. Current Pain and Headache Reports 2007; 11:75-81.

D’Urso A, Serritella E, Tolevski MD, Falisi G,Di Paolo, C. headache and Temporomandibular disorders: Epidemiological assessment. Minerva Stomatological.2016;65(2):85-92
15. Intra-oral Appliances for Headaches and Trigeminal Neuralgia. Clinical policy bulletin aetna ;2018;1-12. http://aetnet.aetna.com/mpa/cpb/600_699/0688.html

16. Türp JC, Komine F, Hugger A. Efficacy of stabilization splints for the management of patients with masticatory muscle pain: a qualitative systematic review. Clin. Oral Investigion $2004 ; 8: 179-195$.

17. Paulo CRC, Ana Corrêa, Lauris JRP, Stuginski Barbosa J Management of painful temporomandibular joint clicking with different intraoral devices and counseling: a controlled Study. J Appl Oral Sci.2015;23: 529-535.

18. Đjorđević Igor, Ana Todorovic, vojkan Lazic. Occlusal appliances - An alternative in temporomandibular disorders pain treatment. Srp. Arh. Celok. Lek. 2019; 541-546.

19. Bruno, MAD, Krymchantowski AV, Amitriptyline and intraoral devices for migraine prevention: A randomized comparative trial. Arq. Neuropsiquiatr.2018; 76:213-218

20. Emshoff R. Clinical factors affecting the outcome of occlusal splint therapy of temporomandibular joint disorders. J. Oral Rehabil.2006;3:393-401.

21. Al-Moraissi, E. A, R Feria, MS AL -Wadeia, ME AL -Sabahie et al Effectiveness of occlusal splint therapy in the management of temporomandibular disorders: network meta-analysis of randomized controlled trials. Int. J. Oral Maxillofac. Surg .2020:49(8):10421056.

22. Dalewski B, Chrus̈ciel-Nogalska M, Frączak B. Occlusal splint versus modified nociceptive trigeminal inhibition splint in bruxism therapy: A randomized, controlled trial using surface electromyography. Aust Dent J. 2015; 60:445-454.

23. Stephen, VE Kamakshi. Occlusal splint treatment in temporomandibular disorder. J. Pain Manag .2013;6:291-295.

24. Zonnenberg, AJJ, Mulder J. The efficacy of a specific stabilization splint. Cranio - J. CraniomandibPract. 2014; 32: 68-74.

25. Jokstad A. The NTI-tss device may be used successfully in the management of bruxism and TMD Evid. Based. Dent.2009;10-23.

26. Azangoo Khiavi H. et al. Efficacy of low-level laser, hard occlusal appliance, and conventional pharmacotherapy in the management of myofascial pain dysfunction syndrome; A preliminary study. J Lasers Med. Sci.2022;11:37-44. 
27. Hasanoglu Erbasar, G. N., Alpaslan C,Eroglu Inan, G. Can an NTI-tss device be effective as a first-line therapy in patients with TMD myofascial pain? J. Oral Rehabil.2017; 44:589-593.

28. Jokstad A, Mo A Krogstad. Clinical comparison between two different splint designs for temporomandibular disorder therapy. Acta Odontol. Scand. 2005; 63: $218-26$ 